\title{
Electronic cigarette use: comparing smokers, vapers, and dual users on characteristics and motivational factors
}

\author{
Claire Schoren ${ }^{1}$, Korin Hummel ${ }^{1}$, Hein de Vries $^{1}$
}

\begin{abstract}
INTRODUCTION This study examined vaping behaviour, precursors of vaping, and motivational differences between smokers, dual users and vapers. The objectives were to assess a) vaping characteristics and reasons for use, b) differences in motivational factors and behavioural precursors associated with e-cigarette use, and c) socio-demographic and motivational factors associated with electronic cigarette use.

METHODS A cross-sectional survey among 259 vapers, 135 smokers, and 83 dual users was conducted in the Netherlands. The questionnaire, based on the I-Change Model, assessed demographics, smoking and vaping behaviour, quit attempt, attitudes towards e-cigarettes, social influences, self-efficacy about not to vape and intention to quit.

RESULTS Vaping e-cigarettes was mostly started for health reasons. Less than $2 \%$ of the vapers had never used conventional cigarettes. Vapers reported most advantages of e-cigarettes whereas smokers were least convinced of them, encountered more modelling of vaping in their social environment and reported higher self-efficacy to control vaping. Older respondents and respondents with low levels of education and low income were more likely to use e-cigarettes instead of conventional cigarettes.

concLusions Although vaping was mostly started for health reasons, the use of the product was not strictly limited to (former) smokers. Health communication for non-smokers is needed to stress potential dangers. Both vapers and non-vapers encounter barriers for using e-cigarettes in difficult situations. If e-cigarettes are used as a smoking cessation tool, more information is needed to help users cope with these situations. For future interventions we recommend to take potential motivational differences among low and high income and educated groups into account.
\end{abstract}

\section{INTRODUCTION}

Since its introduction in 2003, electronic cigarettes (e-cigarettes) are gaining worldwide more and more popularity ${ }^{1-3}$. In the Netherlands, e-cigarette use in the overall Dutch (15 year and older) population rose from $1 \%$ in 2012 to $4.1 \%$ in 2014 , and from $4 \%$ in 2013 to $16 \%$ in 2014 among smokers ${ }^{4,5}$. Advertisements for e-cigarette were not allowed in the Netherlands from January 2008 to June 2012. In August 2013, e-cigarette advertisements began to appear on television ${ }^{6}$. At the time of the conduction of this study, e-cigarettes were classified as a consumer product in the Netherlands. Hence, e-cigarettes could be advertised, no age requirements were set in purchasing the product and the product could be sold in every store.

\section{AFFILIATION}

1 Maastricht University,

Netherlands

CORRESPONDENCE TO

Claire Schoren. Maastricht University, P. Debyeplein 1, 6200 MD Maastricht, Netherlands. Email: c.schoren@ maastrichtuniversity. $\mathrm{nl}$

\section{KEYWORDS}

Smoker, beliefs, public health, e-cigarette, vaping, dual use conventional smokers ${ }^{7-12}$. Commonly reported reasons for using e-cigarettes (also known as vaping) are to quit or reduce smoking regular cigarettes, to reduce feelings of craving, and to reduce health risks caused by smoking ${ }^{4,7,10,13-19}$. Yet, vapers also reveal uncertainty concerning its harmful effects ${ }^{20}$.

Controversy about e-cigarettes remains. Several studies suggest that e-cigarettes may be effective to reduce harm and to support smoking cessation ${ }^{14,21-30}$. In contrast, it is hypothesized that dual users (e-cigarette users who continue smoking conventional cigarettes) are exposed to higher levels of nicotine, become more nicotine dependent, and reduce the chance of cessation ${ }^{11}$. Further, e-cigarette use may lead 
to normalization of e-cigarette use and renormalization of smoking ${ }^{31}$. Additionally, growing popularity and use among adolescents is reported and gateway effects to cigarettes are proposed $^{32-35}$.

Dicey showed that $84 \%$ of the UK e-cigarette users also continued smoking conventional cigarettes, suggesting that motives for using e-cigarettes may be diverse $^{36}$. Whereas social-psychological models have been used to explain various behaviours, studies assessing potential motivational differences between vapers and dual users are relatively scarce, but increasing ${ }^{37-38}$. Yet, such findings are needed to better understand these motives and to optimize health communication about e-cigarettes tailored to the opinions of the various groups of (potential) users ${ }^{39}$.

This study assessed vaping behaviour, behavioural precursors of vaping, smoking and dual use, and motivational differences between smokers, dual users and exclusive vapers (in this paper: vapers) using the I-Change Model. Little information about these groups is available in the Netherlands. Hence, besides assessing attitudes towards vaping, social support and self-efficacy concerning vaping are also important factors, but the latter aspect has not been extensively studied. The main objectives of this study are a) to assess vaping characteristics and reasons for use for both vapers and dual users, b) to identify differences in motivational factors and behavioural precursors related with e-cigarette use between smokers, vapers, and dual users, and c) to gain more insight into the socio-demographic and motivational factors associated with e-cigarette use.

\section{METHODS}

\section{Sample and procedure}

Data was obtained between May and August 2014 by using a Dutch online survey. Inclusion criteria were being a smoker and/or vaper, being between 18 and 65 years old, and being aware of the existence of e-cigarettes. Respondents were recruited via internet by Facebook advertisements and calls on (e-)cigarette related discussion boards. In addition, 200 flyers were distributed among different public places.

The online questionnaire was based on previous research using the I-Change Model and a previous German study ${ }^{40}$. A total of 970 people visited the website. From this group, 49 respondents did not meet the inclusion criteria, 84 respondents did not answer the informed consent question, and 69 respondents answered the informed consent question negatively. This resulted in 768 respondents who started the questionnaire (79\% of the visitors), and a total of 477 complete cases ( $49 \%$ of the visitors and $62 \%$ of the people who actually started the questionnaire). The questionnaire took approximately 30 minutes to complete. Among respondents who completed the questionnaire 20 gift certificates to the value of 25 euro were raffled.

The study was approved by the Medical Ethics Committee of the Atrim-Orbis-Zuyd Hospital (14-N-63).

\section{Theoretical fromework}

The Integrated Model for Change (I-Change model) was used in order to assess relationships with perceived pros and cons of vaping, social modelling and social norms towards vaping, and self-efficacy towards vaping. The I-Change Model assumes three phases in the behavioural change process: awareness (determined by cognizance, knowledge, risk perceptions, and cues to action), motivation (determined by attitudes, social influence beliefs and self-efficacy) and action (determined by self-efficacy, intentions, action planning and plan enactment). Additionally, these phases are influenced by distal preceding factors (such as personality, social environmental factors, and information factors (such as the type and quality of the health communication provided $)^{41-44}$. This study focused on the motivational determinants as specified by the I-Changed Model.

\section{Demographics}

Demographic information was assessed by seven items, pertaining gender, age, nationality, marital status ( $1=$ single; $2=$ in a relationship/cohabiting; $3=$ married; $4=$ divorced; $5=$ widow(er); $6=$ other $)$, and the highest completed level of education ( $1=$ low [no education, primary school, lower vocational education and general secondary education]; $2=$ middle [secondary vocational education and secondary education]; $3=$ high [higher education and university education]). The total amount of monthly income (after the subtraction of taxes) was assessed by three categories ( $1=$ low [up to $€ 1300$ ]; $2=$ middle [ $€ 1300$ to 2400 ]; 3=high [€ 2400 and above]). Employment status was assessed by two categories ( $1=$ Employed [entrepreneur, wage labour, and employment for government]; 2=Unemployed [incapacitated, unemployed, retired, student, and homemaker]).

\section{General smoking/vaping behaviour}

Respondents' general smoking/vaping behaviour was assessed by two items; 'I smoke/vape..' (1=both conventional cigarettes and e-cigarettes; $2=$ solely conventional cigarettes; $3=$ solely e-cigarettes) and 'I..' ( $1=$ first smoked conventional and later began to vape e-cigarettes; $2=$ first vaped e-cigarettes and later began to smoke conventional cigarettes; $3=$ smoke 
conventional cigarettes and never vaped e-cigarettes; 4=vape e-cigarettes and never smoked conventional cigarettes; $5=$ first smoked conventional cigarettes, and have vaped e-cigarettes in-between; $6=$ first vaped e-cigarettes, and have smoked conventional cigarettes in-between).

\section{Smoking behaviour}

Smokers and dual users received three questions regarding their smoking behaviour. These questions assessed the frequency of smoking cigarettes ('How often do you smoke?'), onset ('Since when do you smoke?') and number of cigarettes smoked ('How many cigarettes do you smoke on average per day?').

\section{Vaping behaviour}

Vapers and dual users received five questions regarding their vaping behaviour. These questions assessed the frequency of vaping e-cigarettes ('How often do you vape?'), onset ('Since when do you vape?'), reasons to start ('What was your main reason to start to use the e-cigarette?'), how respondents became familiar with e-cigarettes ('How did you become familiar with the e-cigarette?'), and used cartridges ('I vape..'(nicotine-free versus nicotine-containing cartridges)).

\section{Current quit attempt}

Two items assessed quitting behaviour of conventional cigarettes. First, respondents were asked if they were currently attempting to quit (not smoking for at least 24 hours) conventional cigarettes $(1=\mathrm{no} ; 2=\mathrm{yes})$. Secondly, respondents who were making a quit attempt were asked since when they were making this attempt (open question: number of years/ months/weeks/days).

\section{Attitudes towards e-cigarettes}

Attitudes towards e-cigarettes were assessed by 25 items on a five point Likert scale about perceived advantages and 23 items on perceived disadvantages of e-cigarettes. An overview of the items is depicted in Table 2 (advantages) and Table 3 (disadvantages).

Due to small variances in both constructs, items were dichotomised. Perceived advantages $(a=.93)$ were recoded into either $0=$ totally disagree/disagree/neutral or $1=$ agree/ totally agree. Perceived disadvantages $(a=.92)$ were recoded into either $0=$ totally agree/agree/neutral or $1=$ disagree/totally disagree.

\section{Social Influences}

Social influence was measured by eight social modelling questions and eight social norm questions. First, respondents were asked to indicate whether their partner smoked conventional cigarettes $(0=$ no/non-applicable; $1=y e s)$, and how many of their family members, friends, and colleagues smoked conventional cigarettes $(1=$ all; $2=$ more than half; $3=$ half; $4=$ less than half; $5=$ none; $6=$ non-applicable). The same four questions were asked regarding vaping e-cigarettes.

Due to small variances in both constructs, items were dichotomised into 0 (receiving no modelling, which included 'non-applicable') or 1 (receiving modelling). Scales were calculated by sum scores (modelling conventional cigarettes: $\mathrm{a}$ $=.45$, modelling e-cigarettes: $\mathrm{a}=.56$ ).

Social norm was also assessed by two constructs. 'Would your (partner/family member/friends/ colleagues) prefer that you vape e-cigarettes instead of conventional cigarettes $(a=.95)$ ' and 'Does your (partner/ family member/friends/ colleagues) think vaping e-cigarettes is good? $(a=.68)$ '. Questions were answered on a six point scale (1=totally disagree; $5=$ totally agree; $6=$ non-applicable).

\section{Self-efficacy}

Self-efficacy beliefs about not vaping in potential difficult situations were assessed by 16 items $(a=.95)$ on a five point Likert scale (see Table 4 for an overview). Self-efficacy about not to vape was measured among vapers, dual users and smokers. When assessing self-efficacy, it is custom to ask both participants engaging and not-engaging is a particular behaviour to provide their self-efficacy ratings.

\section{Intention to quit electronic and conventional cigarettes}

The intention to quit electronic and/or conventional cigarettes was assessed by two items. Respondents indicated on a scale from 1 (very weak) to 10 (very strong), how strong they were intending to stop 1) conventional cigarettes and 2) e-cigarettes.

\section{Data analysis}

The three groups (smokers, vapers and dual users) were classified based on how respondents considered themselves according to the first general smoking/vaping behaviour question. General descriptive analyses were performed to describe the whole sample and the three groups. Group differences regarding sample and vape characteristics were identified by performing Chi-Square tests. ANOVA analyses assessed differences between vapers and dual users (see table 1 for included independent variables). Differences between smokers, vapers and dual users were tested by post hoc pairwise comparisons by Tukey HSD tests (see table 2, 3 and 4 for the included independent variables).

Multinomial logistic regression analyses were performed 
to identify associations between socio-demographic variables and the constructs from the I-Change model with respondents' smoking behaviour. In the regression analyses smoking behaviour (either: smoker, vaper or dual user) was used as dependent variable and only included complete cases (see table 5 for the included independent variables). Items that were answered with 'I don't know' were recoded as missing value. In total, 60 of the 477 respondents were excluded from the analyses, because they had a missing value on any of the variables. This resulted in 104 smokers, 240 vapers and 73 dual users included in the regression analyses.

All analyses were conducted with IBM SPSS statistics (version 20).

\section{RESULTS}

\section{Sample description}

Table 1 provides an overview of the sample characteristics. In total 259 (54\%) vapers, 135 (28\%) smokers and 83 (18\%) dual users completed the questionnaire. The questionnaire was completed by 185 men, and respondents had an average age of 37.1 years. Most (95\%) of the respondents were Dutch, $3.6 \%$ had the Belgian nationality and $1.4 \%$ had a different nationality. Vapers more often had a higher income and paid work in comparison to smokers.

Only 5 of the 259 vapers (1.9\%) had never smoked conventional cigarettes. The other 254 vapers indicated that they had smoked conventional cigarettes in the past, but

Table 1. Sample and vaping characteristics.

\begin{tabular}{|c|c|c|c|c|c|}
\hline & Total $(n=477)$ & Smokers $(n=135)$ & Dual users ( $n-83$ ) & Vapers $(n=259)$ & $X^{2}$ \\
\hline Gender $[\%$ male $(N)]$ & $38.8(185)$ & $20.0(27)$ & $32.5(27)$ & $50.6(131)$ & $36.6^{* * *}$ \\
\hline Age [mean (SD)] & $37.1(14.0)$ & $29.0(12.0)$ & $39.1(13.7)$ & $45.9(9.7)$ & NA \\
\hline Marital status & & & & & $48.9^{* * *}$ \\
\hline$[\%$ Single $(N)]$ & $26.8(128)$ & $43.7(59)$ & $28.9(24)$ & $17.4(45)$ & \\
\hline [\% Partner/Cohabiting N)] & $26.6(127)$ & $30.4(41)$ & $24.1(20)$ & $25.5(66)$ & \\
\hline$[\%$ Married $(\mathrm{N})]$ & $37.9(181)$ & $18.5(25)$ & $38.6(32)$ & $47.9(124)$ & \\
\hline$[\%$ Divorced $(\mathrm{N})]$ & $5.5(26)$ & $5.2(7)$ & $3.6(3)$ & $6.2(16)$ & \\
\hline [\% Widow/Widower (N)] & $1.5(7)$ & $0(0)$ & $2.4(2)$ & $1.9(5)$ & \\
\hline$[\%$ Other $(\mathrm{N})]$ & $1.7(8)$ & $2.2(3)$ & $2.4(2)$ & $1.2(3)$ & \\
\hline Educational level; $n=472$ & & & & & NS \\
\hline Low $[\%(N)]$ & $30.2(144)$ & $31.3(42)$ & $31.3(26)$ & $29.8(76)$ & \\
\hline Middle $[\%(N)]$ & $41.7(199)$ & $46.3(62)$ & $45.8(38)$ & $38.8(99)$ & \\
\hline High [\% (N)] & $27.0(129)$ & $22.4(30)$ & $22.9(19)$ & $31.4(80)$ & \\
\hline Income; $n=454$ & & & & & $28.1^{* * *}$ \\
\hline Low $[\%(N)]$ & $24.7(112)$ & $38.8(46)$ & $28.8(23)$ & $16.9(43)$ & \\
\hline Middle $[\%(\mathrm{~N})]$ & $41.2(187)$ & $40.0(48)$ & $43.8(35)$ & $40.9(104)$ & \\
\hline High [\% (N)] & $17.2(78)$ & $12.5(15)$ & $15.0(12)$ & $20.1(51)$ & \\
\hline Refused $[\%(\mathrm{~N})]$ & $17(77)$ & $9.2(11)$ & $12.5(10)$ & $22.0(56)$ & \\
\hline Work situation; $n=465$ & & & & & $28.4^{* * *}$ \\
\hline Working [\% (N)] & $63.4(295)$ & $48.1(64)$ & $55.4(46)$ & $74.3(185)$ & \\
\hline Nonworking [\% (N)] & $36.6(170)$ & $51.9(69)$ & $44.6(37)$ & $25.7(64)$ & \\
\hline Current quit attempt [\% yes $(\mathrm{N})] ; n=472$ & $29.8(142)$ & $10.4(14)$ & $22.9(19)$ & $42.9(109)$ & $46.9^{* * *}$ \\
\hline Vaping frequency; $n=342$ & & & & & $142^{* * *}$ \\
\hline [\% Daily $(\mathrm{N})]$ & 85.7 (293) & NA & $45.8(38)$ & $98.5(255)$ & \\
\hline$[\%$ Daily < weekly $(\mathrm{N})]$ & $7.0(24)$ & NA & $26.5(22)$ & $0.8(2)$ & \\
\hline$[\%$ Weekly < monthly $(\mathrm{N})]$ & $4.1(14)$ & NA & $15.7(13)$ & $0.4(1)$ & \\
\hline$[\%$ Monthly < $(\mathrm{N})]$ & $3.2(11)$ & NA & $12.0(10)$ & $0.4(1)$ & \\
\hline Vaping onset; $n=342$ & & & & & $27.8^{* * *}$ \\
\hline$[\%<1$ month $(\mathrm{N})]$ & $3.2(11)$ & NA & $6.0(5)$ & $2.3(6)$ & \\
\hline$[\% 1<3$ months $(\mathrm{N})]$ & $16.1(55)$ & NA & $27.7(23)$ & $12.4(32)$ & \\
\hline
\end{tabular}




\begin{tabular}{|c|c|c|c|c|c|}
\hline & Total $(n=177)$ & Smokers $(n=135)$ & Dual users ( $n=83)$ & Vapers $(n=259)$ & $X^{2}$ \\
\hline$[\% 3<6$ months $(\mathrm{N})]$ & $21.3(73)$ & NA & $28.9(24)$ & $18.9(49)$ & \\
\hline$[\% 6<12$ months $(\mathrm{N})]$ & $36.8(126)$ & NA & $30.1(25)$ & $39.0(101)$ & \\
\hline$[\% 1<5$ years $(\mathrm{N})]$ & $20.5(70)$ & NA & $7.2(6)$ & $24.7(64)$ & \\
\hline$[\% 5<10$ years $(\mathrm{N})]$ & $2.0(7)$ & NA & $0(0)$ & $2.7(7)$ & \\
\hline Vaping reason to start; $n=342$ & & & & & $68.8^{* * *}$ \\
\hline [\% Less bad for health $(\mathrm{N})]$ & $52.0(178)$ & NA & $26.5(22)$ & $60.2(156)$ & \\
\hline [\% Smoking cessation tool $(\mathrm{N})$ ] & $23.7(81)$ & NA & $25.3(21)$ & $23.2(60)$ & \\
\hline [\% Limit the amount of cigarettes $(\mathrm{N})$ ] & $6.7(23)$ & NA & $21.7(18)$ & $1.9(5)$ & \\
\hline $\begin{array}{l}\text { [\% Smoke where cigarettes are prohibited } \\
(\mathrm{N})]\end{array}$ & $4.1(14)$ & NA & $10.8(9)$ & $1.9(5)$ & \\
\hline$[\%$ better taste $(\mathrm{N})]$ & $2.9(10)$ & NA & $0(0)$ & $3.9(10)$ & \\
\hline$[\%$ Cheaper $(\mathrm{N})]$ & $6.7(23)$ & NA & $10.8(9)$ & $5.4(14)$ & \\
\hline $\begin{array}{l}\text { [\% Replace a certain amount of cigarettes } \\
(\mathrm{N})]\end{array}$ & $.9(3)$ & NA & $1.2(1)$ & $0.8(2)$ & \\
\hline$[\%$ Other reason $(\mathrm{N})]$ & $2.9(10)$ & NA & $3.6(3)$ & $2.7(7)$ & \\
\hline Vaping familiar through; $n=342$ & & & & & NS \\
\hline [\% General practitioner / Practice nurse (N)] & $.3(1)$ & NA & $1.2(1)$ & $0(0)$ & \\
\hline [\% Internet $(\mathrm{N})]$ & $34.5(118)$ & NA & $31.3(26)$ & $35.5(92)$ & \\
\hline$[\%$ TV commercial $(\mathrm{N})]$ & $3.2(11)$ & NA & $4.8(4)$ & $2.7(7)$ & \\
\hline [\% Acquaintances, friends or family $(\mathrm{N})]$ & $53.2(182)$ & NA & $54.2(45)$ & $52.9(137)$ & \\
\hline$[\%$ Advertisements $(\mathrm{N})]$ & $2.6(9)$ & NA & $3.6(3)$ & $2.3(6)$ & \\
\hline$[\%$ Other $(\mathrm{N})]$ & $6.1(21)$ & NA & $4.8(4)$ & $6.6(17)$ & \\
\hline Vaping used cartridges/liquids; $n=342$ & & & & & $15.2^{*}$ \\
\hline [\% Solely nicotine $(\mathrm{N})]$ & $55.6(190)$ & NA & $68.7(57)$ & $51.4(133)$ & \\
\hline$[\%$ Mainly nicotine $(\mathrm{N})]$ & $20.5(70)$ & NA & $12.0(10)$ & $23.2(60)$ & \\
\hline [\% Both nicotine and nicotine free $(\mathrm{N})]$ & $14.6(50)$ & NA & $12.0(10)$ & $15.4(40)$ & \\
\hline [\% Mainly nicotine free $(\mathrm{N})]$ & $4.4(15)$ & NA & $4.8(4)$ & $4.2(11)$ & \\
\hline$[\%$ Solely nicotine free $(\mathrm{N})]$ & $4.1(14)$ & NA & $0(0)$ & $5.4(14)$ & \\
\hline [\% I don't know (N)] & $.9(3)$ & NA & $2.4(2)$ & $0.4(1)$ & \\
\hline
\end{tabular}

${ }^{*} P<.05,{ }^{* *} P<0.01,{ }^{* * *} P<.001$.

successfully quit smoking. Almost $23 \%$ of the dual users and $10.4 \%$ of the smokers indicated to be currently involved in a quit attempt. Yet, additional analyses concerning the quit attempt for both dual users and smokers revealed that these quit attempts were unsuccessful.

Table 1 also shows that vapers were significantly more often daily users and had used e-cigarettes for a longer period of time than dual users. Although group differences were found, the majority among both groups indicated to use mainly or solely nicotine-containing cartridges.

Significant differences in reasons to start e-cigarette use were found as well. Among $60 \%$ of the vapers and $27 \%$ of the dual users, the most frequently mentioned most important reason for use was believing that the product would be better for their health. Using e-cigarettes as smoking cessation tool was indicated to be the second most important reason for use. Dual users indicated 'to limit the amount of conventional cigarettes' as a third important reason for use.

\section{Motivational factors Perceived advantages}

Table 2 shows that there were significant group differences regarding mean scores of agreement on 20 of the 25 statements about advantages of e-cigarettes. Smokers scored lowest on 
Table 2. Differences in perceived advantages about the electronic cigarette; stratified by smoking status.

\begin{tabular}{|c|c|c|c|c|}
\hline & Smokers $(n=135)$ & Dual users ( $n$ - 83) & Vapers $(n=259)$ & Tukey IISD \\
\hline \multicolumn{5}{|l|}{$\begin{array}{l}\text { Perceived advantages; } n=477 \\
\text { Vaping electronic cigarettes... }\end{array}$} \\
\hline Is cheaper than conventional cig. [mean (SD)] & $3.5(.9)$ & $4.2(1.0)$ & $4.2(1.0)$ & $1<2,3^{* * *}$ \\
\hline Is healthier than conventional cig. [mean (SD)] & $3.1(1.1)$ & $4.0(1.1)$ & $4.7(.7)$ & $1<2^{* * *} ; 2<3^{* * *}$ \\
\hline Tastes just as good as conventional cig. [mean (SD)] & $2.1(.9)$ & $2.5(1.3)$ & $3.6(1.5)$ & $1,2<3^{* * *}$ \\
\hline Is cool/tough [mean (SD)] & $1.9(1.0)$ & $1.8(.9)$ & $1.9(1.0)$ & NS \\
\hline Is easy to use [mean (SD)] & $3.2(1.0)$ & $3.5(.9)$ & $3.7(1.1)$ & NS \\
\hline Offers the option to use without nicotine [mean (SD)] & $3.0(1.1)$ & $3.4(1.2)$ & $4.0(1.1)$ & $1<2^{*} ; 2<3^{* * *}$ \\
\hline Is sociable [mean (SD)] & $2.4(1.0)$ & $2.8(1.1)$ & $3.2(1.2)$ & $1<2^{*} ; 2<3^{*}$ \\
\hline Keeps me in a good mood [mean (SD)] & $2.6(1.0)$ & $3.3(1.2)$ & $4.0(1.1)$ & $1<2^{* * *} ; 2<3^{* * *}$ \\
\hline Protects me against withdrawal symptoms [mean (SD)] & $2.7(1.0)$ & $3.5(1.2)$ & $4.2(.9)$ & $1<2^{* * *} ; 2<3^{* * *}$ \\
\hline Is relaxing [mean (SD)] & $2.7(.9)$ & $3.5(1.2)$ & $4.5(.7)$ & $1<2^{* * *} ; 2<3^{* * *}$ \\
\hline Creates less burden to my social environment [mean (SD)] & $3.5(1.0)$ & $4.1(.9)$ & $4.6(.8)$ & $1<2^{* * *} ; 2<3^{* * *}$ \\
\hline Is allowed everywhere [mean (SD)] & $3.2(1.1)$ & $3.4(1.1)$ & $3.4(1.2)$ & NS \\
\hline Helps me to limit my smoke-behaviour [mean (SD)] & $3.0(1.1)$ & $4.2(1.0)$ & $4.6(.8)$ & $1<2^{* * *} ; 2<3 * * *$ \\
\hline Satisfies my need for nicotine [mean (SD)] & $2.6(.9)$ & $3.6(1.2)$ & $4.3(.9)$ & $1<2^{* * *} ; 2<3 * * *$ \\
\hline Helps me not to smoke conventional cig. [mean (SD)] & $2.5(.9)$ & $3.4(1.2)$ & $4.8(.6)$ & $1<2^{* * *} ; 2<3^{* * *}$ \\
\hline Is a better smoking cessation tool [mean (SD)] & $2.6(1.0)$ & $3.7(1.1)$ & $4.7(.7)$ & $1<2^{* * *} ; 2<3^{* * *}$ \\
\hline Damages my health less than conventional cig. [mean (SD)] & $3.1(1.0)$ & $4.0(1.0)$ & $4.7(.7)$ & $1<2^{* * *} ; 2<3^{* * *}$ \\
\hline Is odourless [mean (SD)] & $3.4(1.1)$ & $4.3(.9)$ & $4.6(.7)$ & $1<2^{* * *} ; 2<3 * *$ \\
\hline Ensures me not inhaling tar/carbon monoxide [mean (SD)] & $3.5(.9)$ & $4.4(.9)$ & $4.8(.5)$ & $1<2^{* * *} ; 2<3^{* * *}$ \\
\hline Ensures, at long-term, for less need for nicotine [mean (SD)] & $2.8(.9)$ & $3.6(1.1)$ & $4.4(.9)$ & $1<2^{* * *} ; 2<3^{* * *}$ \\
\hline Ensures me more air [mean (SD)] & $2.8(.9)$ & $3.7(1.2)$ & $4.6(.7)$ & $1<2^{* * *} ; 2<3^{* * *}$ \\
\hline Ensures me more energy [mean (SD)] & $2.8(.8)$ & $3.4(1.2)$ & $4.5(.8)$ & $1<2^{* * *} ; 2<3^{* * *}$ \\
\hline Has no fire risk [mean (SD)] & $3.4(.9)$ & $3.8(1.1)$ & $4.3(1.0)$ & $1<2^{*} ; 2<3^{* * *}$ \\
\hline Will not give you yellow teeth / brown fingers [mean (SD)] & $3.5(.9)$ & $4.1(.9)$ & $4.8(.6)$ & $1<2^{* * *} ; 2<3^{* * *}$ \\
\hline Is easy, because it is compact [mean (SD)] & $3.1(1.0)$ & $3.5(1.0)$ & $3.8(1.2)$ & $1<2^{* * *} ; 2<3^{* * *}$ \\
\hline
\end{tabular}

Cig: Cigarette

Measured on a 5-point scale: 1 = completely disagree, 5 = completely agree

${ }^{*} \mathrm{P}<0.05,{ }^{* *} \mathrm{P}<0.01,{ }^{* * *} \mathrm{P}<.001$.

the 20 items and were least convinced of the product, while vapers scored the highest and were most convinced. Smokers perceived vaping, in comparison to both groups, significantly less often to be cheaper than smoking conventional cigarettes. Vapers perceived vaping significantly more often to be just as tasty as smoking, in comparison to both other groups.

\section{Perceived disadvantages}

Table 3 shows that there were significant group differences regarding mean scores of agreement on 14 of the 23 statements about disadvantages of e-cigarettes. The highest scores on the disadvantages scale were found among the smokers while vapers reported the lowest scores.

Smokers perceived vaping significantly more often as costly, time-consuming, unhandy, causing nausea, dizziness, and/or mouth and throat irritations. Vapers perceived vaping, compared to both other groups, significantly less often to be shifting the nicotine-dependence and leading to increases in toxic substances in their body. Vapers perceived vaping, in comparison to smokers, significantly less often to be addictive.

\section{Social influences}

An overview of the social influence items and the differences between the three groups can be found in Table 4 .

Significant differences between the groups were found on all eight items of the two social norm constructs. Vapers scored the highest, indicating that their social environment had a more positive attitude towards e-cigarettes compared to the smokers who scored the lowest.

Furthermore, vapers experienced significantly less social modelling for the conventional cigarette from their partner, family members, and friends compared to dual users and 
Table 3. Differences in perceived disadvantages about the electronic cigarette; stratified by smoking status.

\begin{tabular}{|c|c|c|c|c|}
\hline & $\begin{array}{l}\text { Smokers } \\
(\mathbf{n}=\mathbf{1 3 5})\end{array}$ & $\begin{array}{l}\text { Dual users } \\
\quad(n=83)\end{array}$ & $\begin{array}{l}\text { Vapers } \\
(n=259)\end{array}$ & Tukey IISD \\
\hline \multicolumn{5}{|l|}{$\begin{array}{l}\text { Perceived disadvantages; } n=477 \\
\text { Vaping electronic cigarettes... }\end{array}$} \\
\hline Can damage the health of my social environment [mean (SD)] & $3.4(.8)$ & $3.9(.8)$ & $4.2(.9)$ & $1<2^{* * *} ; 2<3^{*}$ \\
\hline Is difficult/unhandy/complex [mean (SD)] & $3.1(.9)$ & $3.8(1.0)$ & $4.1(.9)$ & $1<2^{* * *} ; 2<3^{* *}$ \\
\hline Is unhealthy [mean (SD)] & $2.9(.9)$ & $3.4(1.0)$ & $4.0(1.0)$ & $1<2^{* *} ; 2<3^{* * *}$ \\
\hline Shifts my nicotine-dependence [mean (SD)] & $2.9(1.0)$ & $3.0(1.0)$ & $3.3(1.2)$ & $1,2<3^{*}$ \\
\hline Makes me, in ratio, smoke more than conventional cig. [mean (SD)] & $3.0(1.0)$ & $3.5(1.0)$ & $4.0(1.1)$ & $1<2^{* *} ; 2<3^{* *}$ \\
\hline Costs a lot of money [mean (SD)] & $3.1(.8)$ & $3.8(1.0)$ & $3.9(1.0)$ & $1<2,3^{* * *}$ \\
\hline Is a burden to my environment [mean (SD)] & $3.5(.8)$ & $4.2(.7)$ & $4.5(.7)$ & $1<2^{* * *} ; 2<3^{* * *}$ \\
\hline Is shameful [mean (SD)] & $3.2(.8)$ & $3.9(.9)$ & $4.4(.9)$ & $1<2^{* * *} ; 2<3^{* * *}$ \\
\hline Costs a lot of time [mean (SD)] & $3.2(.8)$ & $3.9(.9)$ & $4.2(1.0)$ & $1<2,3^{* * *}$ \\
\hline Is addictive [mean (SD)] & $2.9(.8)$ & $3.2(1.0)$ & $3.2(1.1)$ & $1<3^{* *}$ \\
\hline Leads to toxic substances in my body [mean (SD)] & $3.0(.9)$ & $3.2(1.0)$ & $3.7(1.1)$ & $1,2<3^{* *}$ \\
\hline Makes it difficult to quit, because of the 'smoke-movement' [mean (SD)] & $2.5(.9)$ & $3.3(1.2)$ & $4.2(1.0)$ & $1<2^{* * *} ; 2<3^{* * *}$ \\
\hline Leads to health risks at long term [mean (SD)] & $2.7(.8)$ & $3.1(.8)$ & $3.5(.9)$ & $1<2^{* * *} ; 2<3^{* *}$ \\
\hline Will encourage smoking conventional cig. [mean (SD)] & $3.0(1.0)$ & $4.1(.9)$ & $4.7(.6)$ & $1<2^{* * *} ; 2<3^{* * *}$ \\
\hline Does not work as a smoking cessation tool [mean (SD)] & $2.6(.9)$ & $3.6(1.1)$ & $4.5(.9)$ & $1<2^{* * *} ; 2<3^{* * *}$ \\
\hline Creates new conventional smokers [mean (SD)] & $2.8(.8)$ & $3.8(1.1)$ & $4.6(.7)$ & $1<2^{* * *} ; 2<3^{* * *}$ \\
\hline Is unhandy: not a lot of stores sell the equipment [mean (SD)] & $2.8(.9)$ & $3.7(1.0)$ & $3.9(1.2)$ & $1<2,3^{* * *}$ \\
\hline Is expensive in first purchase [mean (SD)] & $2.4(.8)$ & $3.0(1.1)$ & $3.2(1.2)$ & $1<2,3^{* *}$ \\
\hline Leads to nausea, dizziness and/or mouth and throat irritations [mean (SD)] & $2.8(.8)$ & $3.2(1.0)$ & $3.5(1.1)$ & $1<2,3^{* *}$ \\
\hline Leads to more vaporizing, to get the same amount of nicotine [mean (SD)] & $2.7(.7)$ & $3.2(1.1)$ & $3.8(1.1)$ & $1<2^{* * *} ; 2<3^{* * *}$ \\
\hline Always requires two e-cig.: battery charge and defects [mean (SD)] & $2.7(.8)$ & $2.7(1.1)$ & $2.5(1.2)$ & NS \\
\hline Increase the change I will start smoking conventional cig. [mean (SD)] & $2.8(.9)$ & $3.9(1.1)$ & $4.8(.6)$ & $1<2^{* * *} ; 2<3^{* * *}$ \\
\hline Will lead to more acceptance of conventional cig. [mean (SD)] & $2.9(1.0)$ & $4.0(.9)$ & $4.7(.7)$ & $1<2^{* * *} ; 2<3^{* * *}$ \\
\hline
\end{tabular}

Cig: Cigarette, E-cig: Electronic cigarette

Measured on a 5-point scale: 1 = completely agree, 5 = completely disagree

${ }^{*} P<.05,{ }^{* *} P<0.01,{ }^{* * *} P<.001$.

smokers. Also, the vapers experienced less modelling for the conventional cigarette from their colleagues compared to the smoker group. In all four items regarding social modelling for e-cigarettes, smokers scored lower in comparison to the dual and vapers.

\section{Self-efficacy}

Table 4 illustrates significant group differences on four of the 16 self-efficacy items. Vapers indicated to have the highest selfefficacy, whereas smokers scored the lowest.

Vapers indicated to be significantly more confident to manage not to vape when they see someone vaping, when they are sad, stressed, tensed, when they crave for nicotine, or wake up in the morning, compared to smokers. Vapers indicated to be significantly more confident not to vape when an e-cigarette is offered, when they miss the flavour of conventional cigarettes, and when they just ate.

Smokers indicated to be significantly less confident not to vape when they do not know how to act, when they are bored, and when they had a break from work.

Intention to quit conventional cigarettes. Smokers had a significantly higher intention to quit conventional cigarettes $(\mathrm{M}=6.2)$ than dual users $(\mathrm{M}=4.9 ;(\mathrm{F}=16.2 ; \mathrm{p}<0.001)$.

\section{Factors associated with smoking/vaping behaviour}

Table 5 presents the results of the multinomial regression analyses (N.B. results can differ from our ANOVA analysis due to possible suppressor effects, i.e. the regression analysis corrected the correlations with other included variables). Results revealed that young respondents were more likely to smoke or to smoke in combination with vaping instead of solely vaping e-cigarettes. Older respondents were on the other hand more likely to be dual users instead of smokers. Furthermore, respondents with a higher level of education or higher income 


\section{Research Paper}

Table 4. Differences regarding social Influence and self efficacy items; stratified by smoking behaviour.

Social Norm
My ... prefer(s) me vaping electronic cigarettes instead of smoking
conventional cigarettes:

Partner [mean (SD)]; $\mathrm{n}=350$

Family [mean (SD)]; $n=442$

Friends [mean (SD)]; $\mathrm{n}=439$

Colleagues [mean (SD)]; $\mathrm{n}=346$

My ... think(s) vaping electronic cigarettes is good:

Partner [mean (SD)]; $\mathrm{n}=356$

Family [mean (SD)]; $n=445$

Friends [mean (SD)]; $\mathrm{n}=449$

Colleagues [mean (SD)]; $n=354$

\section{Modelling; $n=477$}

My partner smokes conventional cigarettes [mean (SD)]

How many of your .... smoke conventional cigarettes?

Family members [mean (SD)]

Friends [mean (SD)]

Colleagues [mean (SD)]

My partner vapes electronic cigarettes [mean (SD)]

How many of your .... vape electronic cigarettes?

Family members [mean (SD)]

Friends [mean (SD)]

Colleagues [mean (SD)]

\section{Self-efficacy; $n=477$}

I think I will manage not to vape when... ${ }^{A}$

I am at a party [mean (SD)]

I drink alcohol [mean (SD)]

I am frustrated or mad [mean (SD)]

I see someone vaping [mean (SD)]

I see someone smoke [mean (SD)]

I do not know how to act [mean (SD)]

I am bored [mean (SD)]

I am sad [mean (SD)]

I am stressed or tensed [mean (SD)]

Someone offers me an electronic cigarette [mean (SD)]

I crave for nicotine [mean (SD)]

I get up in the morning [mean (SD)]

I drink coffee or tea [mean (SD)]

I miss the flavour of a conventional cigarettes [mean (SD)]

I just ate [mean (SD)]

I am on break [mean (SD)]

Intention to quit

Conventional cigarettes [mean (SD)]

Electronic cigarettes [mean (SD)]

$\begin{array}{ll}2.3(1.1) & 3.7(1.3) \\ 2.6(1.1) & 3.8(1.2) \\ 2.3(1.0) & 3.3(1.2) \\ 2.3(.9) & 3.2(1.2) \\ 2.7(1.0) & 3.8(1.0) \\ 2.8(.9) & 3.7(1.0) \\ 2.9(.9) & 3.7(1.0) \\ 2.8(.9) & 3.6(.9)\end{array}$

$4.3(1.2)$

$1<2^{* * *} ; 2<3^{* *}$

$4.4(1.1) \quad 1<2^{* * *} ; 2<3^{* * *}$

$4.1(1.1) \quad 1<2^{* * *} ; 2<3^{* * *}$

$4.1(1.0) \quad 1<2^{* * *} ; 2<3^{* * *}$

$4.5(.9) \quad 1<2^{* * *} ; 2<3^{* * *}$

$4.4(.8) \quad 1<2^{* * *} ; 2<3^{* * *}$

$4.4(.8) \quad 1<2^{* * *} ; 2<3^{* * *}$

$4.3(.8) \quad 1<2^{* * *} ; 2<3^{* * *}$

$.38(.5)$

$.41(.5)$

$.2(.4)$

$3<1,2^{* * *}$

$2.49(1.1) \quad 2.3(1.0)$

$2.0(.9)$

$3<1,2 *$

$3.3(1.1) \quad 3.0(1.2)$

$2.5(1.0)$

$2.4(1.1)$

$2.3(1.2)$

$2.0(1.0)$

$.0(.1)$

$.2(.4)$

$.3(.4)$

$3<1,2^{* *}$

$3<1^{* *}$

$1<2,3^{* * *}$

$1.2(.4)$

$1.5(.6)$

$1.7(.9)$

$1<2,3 * *$

$1.2(.4)$

$1.8(.7)$

$1.9(.8)$

$1<2,3^{* * *}$

$1.3(.5)$

1.5 (.7)

$N=135$

$N=83$

$1.6(.8)$

$1<2^{* * *} ; 2<3^{* * *}$

$2.8(1.3)$

$3.3(1.3)$

$N=259$

$2.5(1.3)$

$3.0(1.4)$

$3.8(1.3)$

$1<2^{*} ; 2<3^{* *}$

$2.3(1.2)$

2.7 (1.3)

$3.5(1.4)$

$1<2^{* *} ; 2<3^{*}$

$3.0(1.2)$

$3.3(1.2)$

$3.2(1.4)$

$1<2^{*} ; 2<3^{*}$

2.8 (1.2)

$3.2(1.3)$

$3.6(1.3)$

$1<3^{* * *}$

$2.9(1.2)$

$3.5(1.2)$

$3.8(1.3)$

$1<2 * ; 2<3^{* *}$

2.7 (1.2)

3. 1 (1.2)

$3.8(1.2)$

$1<2,3^{* *}$

$1<2,3^{*}$

$1<3 * * *$

$1<3 * * *$

$2.3(1.1)$

$2.9(1.2)$

$3.2(1.3)$

$3.0(1.3)$

$3.5(1.2)$

$2.8(1.3)$

$1,2<3^{*}$

$1<3^{*}$

$1<3^{* *}$

NS

$3.1(1.2) \quad 3.2(1.3)$

$3.4(1.3)$

$1,2<3^{*}$

$1,2<3^{*}$

$1<2,3^{*}$

$3.0(1.2)$

$3.5(1.4)$

$3.2(1.3)$

$3.0(1.3)$

$6.2(2.5)$

$4.9(2.3)$

NA

$1<2 * * *$

NA

$4.4(2.9)$

$3.9(2.8)$

NS

${ }^{A}$ Measured on a 5-point scale: $1=$ definitely not, 5 = definitely

${ }^{*} P<.05,{ }^{* *} P<0.01,{ }^{* * *} P<.001$. 
Table 5. Multinomial logistic regression analyses; associations of socio-demographics and motivational factors with smoking behaviour.

\begin{tabular}{|c|c|c|c|c|c|c|c|c|c|}
\hline & & Smoke & & & Jual use & & & Dual use & \\
\hline & OR & & & OR & & $\% \mathrm{CI}$ & OR & & $\% \mathrm{CI}$ \\
\hline & & Lower & Upper & & Lower & Upper & & Lower & Upper \\
\hline Gender & 1.96 & .57 & 6.73 & 1.91 & .97 & 3.75 & .97 & .30 & 3.15 \\
\hline Age & $.91 * * *$ & .87 & .96 & $.96^{*}$ & .93 & .99 & $1.06^{*}$ & 1.01 & 1.10 \\
\hline Level of education & $3.16^{* *}$ & 1.38 & 7.22 & 1.28 & .82 & 1.99 & $40^{*}$ & .19 & .87 \\
\hline Work situation & 1.87 & .54 & 6.47 & 2.03 & .97 & 4.26 & 1.08 & .35 & 3.36 \\
\hline Income & $2.08^{*}$ & 1.00 & 4.32 & 1.35 & .93 & 2.00 & .65 & .32 & 1.30 \\
\hline Perceived advantages ${ }^{c}$ & $.71^{* * *}$ & .62 & .82 & $87^{* * *}$ & .80 & .94 & $1.21^{* *}$ & 1.08 & 1.37 \\
\hline Perceived disadvantages $^{c}$ & $.74^{* * *}$ & .66 & .84 & .95 & .87 & 1.04 & $1.28 * * *$ & 1.16 & 1.42 \\
\hline Self-efficacy & $.44^{*}$ & .23 & .84 & $.66^{*}$ & .46 & .95 & 1.48 & .83 & 2.63 \\
\hline $\begin{array}{l}\text { Social norm electronic cigarettes } \\
\text { instead of conventional cigarettes }\end{array}$ & .64 & .32 & 1.27 & .96 & .65 & 1.42 & 1.51 & .80 & 2.83 \\
\hline $\begin{array}{l}\text { Social norm electronic cigarettes } \\
\text { general }\end{array}$ & .62 & .30 & 1.25 & .61 & .37 & 1.01 & 1.00 & .54 & 1.83 \\
\hline Modelling conventional cigarettes ${ }^{c}$ & 1.50 & .78 & 2.89 & $1.55^{*}$ & 1.08 & 2.24 & 1.03 & .56 & 1.93 \\
\hline Modelling electronic cigarettes ${ }^{c}$ & $.35 * *$ & .20 & .63 & .93 & 69 & 1.26 & $2.65^{* * *}$ & 1.54 & 4.57 \\
\hline
\end{tabular}

$R^{2}=.78$

${ }^{A}$ Vapers are the reference category ${ }^{B}$ Smokers are the reference category ${ }^{C}$ Variables in scale are dichotomised

${ }^{*} \mathrm{P}<.05^{* *} \mathrm{p}<.011^{* * *} \mathrm{P}<.001$

levels were more likely to smoke cigarettes than solely vape e-cigarettes. Respondents with low levels of education were more likely to be dual users instead of smokers.

Respondents who perceived fewer advantages of e-cigarettes were more likely to be smokers or dual users than vapers. Respondents who indicated to perceive more advantages were more likely to be dual users than smokers. Similar results were found for the perceived disadvantages: respondents who indicated to perceive more disadvantages were more likely to be smokers than vapers. People who perceived fewer disadvantages were more likely to be dual users than smokers. People with lower self-efficacy values were more likely to be either smokers or dual users.

Finally, people who received more social modelling for conventional cigarettes were more likely to be dual users instead of vapers. People who received less social modelling for e-cigarettes were more likely to be smokers than vapers. People who perceived more social modelling were more likely to be dual users than smokers.

\section{DISCUSSION}

This study assessed vaping characteristics, main reasons for use, differences in motivational factors between smokers, vapers, and dual users and factors associated with e-cigarette use.

Less than $2 \%$ of the vapers had never used conventional cigarettes. Although a small group, this figure shows that e-cigarette use is not strictly limited to smokers, and may thus also be a new (risk) behaviour for non-smokers, in particular youngsters. This was also found in a review of McMillen with corresponding risks such as primary nicotine addiction and renormalization of tobacco use ${ }^{45}$. Awareness of e-cigarette advertisements, which increased sharply in the Netherlands since its introduction in 2013, may also influence non-smokers' perception of e-cigarettes ${ }^{6}$. Hence, monitoring of e-cigarette use and its effects is important to assess potential health impact and potential shifts in user groups, in particular as recent studies in the US suggest that adolescents and young adults who never have used cigarettes but use e-cigarettes may develop positive attitudes towards smoking ${ }^{35,46,47}$.

Vapers used e-cigarettes more often $(98.5 \%)$ on a daily basis compared to dual users (45.8\%), which is congruent with other studies who found group differences in frequency of use $\mathrm{e}^{20,48}$. Brown and colleagues proposed that these findings might be a reflection of vapers escalating their use following cessation in the assumption that e-cigarettes are an effective 
nicotine replacement device ${ }^{20}$. Nicotine-free cartridges were less popular than nicotine-containing cartridges among both groups. This is in line with previous studies, and illustrates the importance of nicotine as an ingredient of the product and e-cigarette users' addiction to nicotine $\mathrm{e}^{8,16,17,49,50}$.

The two most commonly mentioned reasons for using e-cigarettes were that the product was considered as less detrimental for one's health and served as a smoking cessation tool. Among dual users, a third important reason was to limit the number of cigarettes. Similar reasons, albeit without the distinction between smokers, vapers and dual users, have been reported elsewhere as well ${ }^{4,7,8,17,20}$.

Concerning motivational factors, we found that vapers reported most advantages of e-cigarettes whereas smokers were least convinced of them. Similarly, vapers reported the fewest disadvantages of e-cigarettes, and smokers the most. Similar results were found by Rüther and colleagues ${ }^{40}$. Dawkins and colleagues illustrated that vapers who were ex-smokers reported (compared to dual users) significantly more often that e-cigarettes helped them to stop smoking, that they were extremely satisfied with the product, that e-cigarettes reduced craving levels, improved their breathing and were experienced as healthier than smoking cigarettes ${ }^{15}$.

Almost all vapers (98\%) indicated that they used to smoke conventional cigarettes in the past, but successfully stopped smoking. Etter and Bullen also found that most e-cigarette users were former smokers and used the device to assist quitting $^{26}$. Despite previous reviews suggesting positive effects of e-cigarettes in supporting smokers to quit, a recent metaanalysis showed that the odds of quitting cigarettes were $28 \%$ lower among e-cigarette users compared to non-e-cigarette users $^{51-52}$. These contrasting findings illustrate that e-cigarettes may not (always) be beneficial (despite their beliefs that they might be helpful in quitting regular cigarettes). They may at least not help all smokers to quit smoking, and these contrasting findings suggest a need for analysing for which smokers they may or may not work. Well balanced health communication messages are needed in order to help smokers who want to quit, to make an informed decision concerning the most optimal quit method.

Concerning social influences we found that the overall perceived social norm (to what extent does the environment think positively about e-cigarettes), was the highest among vapers and lowest among smokers. Vapers experienced in their social environment less modelling for conventional cigarettes in comparison to dual users and smokers. Yet, vapers experienced more modelling for e-cigarettes. Again, similar results were found in the study of Rüther and colleagues ${ }^{40}$.
Pokhrel and colleagues also acknowledge the role of social influences and state that social influence may be an important barrier for cigarette smokers trying to substitute cigarettes with e-cigarettes ${ }^{53}$.

Smokers were least self-efficacious not to use e-cigarettes in difficult situations. These situations may also be the situations that they encounter as difficult to refrain from smoking in general, suggesting that increasing self-efficacy to cope with urges from smoking for this group may be pivotal. Furthermore, the results among vapers also show situations in which vapers may be less likely to use e-cigarettes. These concern situations that may also result in increased risks for relapsing into smoking, such as when at a party and seeing someone smoking. Our results support earlier findings from a study conducted in Germany and illustrate that if smokers want to use vaping as a cessation tool, they also may need to be informed about potential barriers and how to overcome them $^{40}$. Dual user scores on motivational items were often between those of smokers and vapers. Social support for e-cigarette use among this group, however, was similar as for vapers and significantly higher than for smokers. It thus seems that dual users are supported by others, but not yet fully convinced of potential advantages of e-cigarette use. As our research was cross-sectional we cannot identify the most important factors predicting relapse to full cigarette smoking or full e-cigarette use. Longitudinal research for this group is thus recommended. Most of the items identified appeared to be salient items, implying that these are also relevant items for future health communication messages to guide dual users into smoking cessation.

Our regression analysis revealed that older respondents and respondents with low levels of education and low income were more likely to use e-cigarettes instead of cigarettes. Furthermore, people who perceived more advantages, less disadvantages or more social modelling for e-cigarettes were more likely to be vapers instead of smokers. These results underline the importance of these motivational factors in e-cigarette use behaviour, but also prompt for additional research to understand why e-cigarettes are more appealing to lower educated smokers. Although these factors may predict e-cigarette use, this conclusion cannot be drawn from our cross-sectional study, and more longitudinal research is needed to grasp this process better. With an explained variance of $78 \%$, the I-Change Model illustrates to fit the data well.

\section{Strengths and limitations}

The explorative, theory-driven nature of the study can be considered as one of it its important strengths. The 
distinction between vapers and dual users is not often made. Further, the study examined a broad range of motivational determinants associated with e-cigarette use. These insights offer the opportunity to optimize health communication about e-cigarettes by providing tailored messages to the opinions of the various groups of (potential) users.

This study however also is subject to some limitations. First, most vapers were recruited through online forums. This may have led to an overrepresentation of dedicated users, who may be more positive towards e-cigarette use than non-responders. Also, the provided incentives to respondents could have biased the research sample. Second, as the goal of this study was to study motivational differences between the study groups and not to study e-cigarette prevalence, no conclusions about e-cigarette use on a population level can be drawn from this study. Third, the sample size, especially among dual users and smokers was modest. The sample size was also affected by a non-completion rate of almost $40 \%$, probably caused by the length of the questionnaire. Fourth, we only focused on motivational factors influencing e-cigarette use. Potential other factors of influence (e.g. advertisements of the product or influences of the media or other external factors) are not examined is this study. Fifth, our results are cross-sectional, so no inferences about factors predicting e-cigarette use can be made. Finally, we did not assess pre-motivational and post-motivational factors. Yet, assessing factors that determine awareness of e-cigarette use are also needed as well as studies targeting post-motivational factors such as action planning (preparatory and coping plans) and plan enactment. For instance, a recent study on smoking cessation demonstrated that smokers' intentions to quit were to a large extent explained by action plans and plan enactment ${ }^{42}$.

\section{CONCLUSIONS}

First, our results showed that almost all vapers were former smokers, but that there was a small group who indicated that e-cigarette use is not strictly limited to smokers, and who may need further attention. For future research it is recommended to investigate potential shifts in attitudes towards smoking conventional cigarettes in this group. Also health communication methods are needed to stress the potential dangers of e-cigarettes for never smokers.

Second, e-cigarettes were mainly used for health-related reasons (e.g. less detrimental for one's health, to quit smoking or to limit the amount of cigarettes smoked).

Third, almost all vapers had smoked conventional cigarettes in the past, indicating that e-cigarettes could play a role in the smoking cessation process. As findings concerning the effectiveness or e-cigarette use are conflicting, we suggest to investigate for which smokers e-cigarettes may or may not work in quitting conventional cigarettes.

Fourth, our self-efficacy results show that all three groups may encounter barriers for using e-cigarettes successfully. If e-cigarettes are used as a smoking cessation tool, vapers may profit from more personalized feedback on how to use e-cigarettes and how to cope with difficult situations in order to prevent relapse to regular cigarettes smoking.

Fifth, our results reveal that older respondents and respondents with low levels of education and low income were more likely to use e-cigarettes instead of cigarettes. Additional research to understand why e-cigarettes are more appealing to lower income and lower educated groups is recommended. It is also recommended that future developed interventions or campaigns take these potential motivational differences among these low and high income and educated groups into account.

\section{REFERENCES}

1. Society AC: Electronic Cigarettes (E-Cigarettes). CA: A Cancer Journal for Clinicals [2014], [64]:[3]:169-70.

2. Buisman R, Croes, E: Factsheet Elektronische Sigaretten (E-sigaretten). Trimbos-instituut, Utrecht [2014]. Available at: http://www.trimbos.nl/ /media/Nieuws\%20en\%20Persberichten/ AF1285\%20070414\%20factsheet\%20e-sigaret.ashx. (accessed 30 April 2015)

3. Rijksinstituut voor Volksgezondheid en Milieum (RIVM): E-sigaretten Factsheet. Bilthoven: RIVM [2014]. Available at: http:// www.rivm.nl/dsresource?type $=$ pdf\&disposition $=$ inline\&objectid $=r$ ivmp:242776\&versionid $=\&$ subobjectname $=$. $($ accessed 30 April 2015)

4. Hummel K, Hoving C, Nagelhout GE, Vries Hd, Putter Bvd, Candel MJJM, et al.: Prevalence and reasons for use of electronic cigarettes among smokers: Findings from the International Tobacco Control (ICT) Netherlands Survey. International Journal of Drugs Policy [2015].

5. Verdurmen J, Monshouwer, K., Laar, van, M: Factsheet Continu Onderzoek Rookgewoonten 2014 [Factsheet]. Trimbos Instituut, Utrecht: Trimbos instituut [2014].Available at: https://assets. trimbos.nl/docs/21388531-6303-48f7-9a47-51898fb427df.pdf (accessed 3 August 2016)

6. Nagelhout GE, Heijndijk SM, Cummings KM, Willemsen MC, van den Putte B, Heckman BW, et al.: E-cigarette advertisements, and associations with the use of e-cigarettes and disapproval or quitting of smoking: Findings from the International Tobacco Control (ITC) Netherlands Survey. International Journal of Drug Policy [2016], [29]:73-79.

7. Etter JF: Electronic cigarettes: a survey of users. BMC public health [2010], [10]:[231].

8. Farsalinos KE, Romagna G, Tsiapras D, Kyrzopoulos S, Voudris V: Characteristics, perceived side effects and benefits of electronic cigarette use: a worldwide survey of more than 19,000 consumers. International journal of environmental research and public health [2014], [11]:[4]:4356-73. 
doi: 10.3390/ijerph110404356.

9. McQueen A, Tower S, Sumner W: Interviews with "vapers": Implications for future research with electronic cigarettes. Nicotine \& Tobacco Research [2011], [13]:[9]:860-7.

10. Pepper JK, Brewer NT: Electronic nicotine delivery system (electronic cigarette) awareness, use, reactions and beliefs: a systematic review. Tobacco control [2013], [tobaccocontrol-2013-051122]. doi: 10.1136/tobaccocontrol-2013-051122.

11. Ramo DE, Young-Wolff KC, Prochaska JJ: Prevalence and correlates of electronic-cigarette use in young adults: Findings from three studies over five years. Addictive behaviors [2014], [41C]:[142-7].

12. Regan AK, Promoff G, Dube SR, Arrazola R: Electronic nicotine delivery systems: adult use and awareness of the 'e-cigarette' in the USA. Tobacco control [2013], [22]:[1]:19-23.

13. Adkison SE, O'Connor RJ, Bansal-Travers M, Hyland A, Borland R, Yong $\mathrm{HH}$, et al.: Electronic nicotine delivery systems: international tobacco control four-country survey. American journal of preventive medicine [2013], [44]:[3]:207-15. doi: 10.1136/tobaccocontrol-2011-050044.

14. Adriaens K, Van Gucht D, Declerck P, Baeyens F: Effectiveness of the Electronic Cigarette: An Eight-Week Flemish Study with SixMonth Follow-up on Smoking Reduction, Craving and Experienced Benefits and Complaints. International journal of environmental research and public health [2014], [11]:[11]:11220-48. doi: 10.3390/ijerph111111220.

15. Dawkins L, Turner J, Roberts A, Soar K: 'Vaping' profiles and preferences: an online survey of electronic cigarette users. Addiction (Abingdon, England) [2013], [108]:[6]:1115-25.

doi: 10.1111 /add.12150.

16. Etter JF, Bullen C: Electronic cigarette: users profile, utilization, satisfaction and perceived efficacy. Addiction (Abingdon, England) [2011], [106]:[11]:2017-28. doi: 10.1111/j.1360-0443.2011.03505.x.

17. Goniewicz ML, Lingas EO, Hajek P: Patterns of electronic cigarette use and user beliefs about their safety and benefits: an Internet survey. Drug and alcohol review [2013], [32]:[2\}:133-40. doi: 10.1111/j.1465-3362.2012.00512.x.

18. Pepper JK, Ribisl KM, Emery SL, Brewer NT: Reasons for starting and stopping electronic cigarette use. International journal of environmental research and public health [2014]. [11]:[10]:10345-61. doi: 10.3390/ijerph111010345.

19. Steinberg MB, Zimmermann MH, Delnevo CD, Lewis MJ, Shukla P, Coups EJ, et al.: E-Cigarette Versus Nicotine Inhaler: Comparing the Perceptions and Experiences of Inhaled Nicotine Devices. Journal of general internal medicine [2014], [29]:[11]:1444-50. doi: 10.1007/s11606-014-2889-7.

20. Brown J, West R, Beard E, Michie S, Shahab L, McNeill A: Prevalence and characteristics of e-cigarette users in Great Britain: Findings from a general population survey of smokers. Addictive behaviors [2014], [39]:[6]:1120-5. doi: 10.1016/j.addbeh.2014.03.009

21. Biener L, Hargraves JL: A Longitudinal Study of Electronic Cigarette Use in a Population-based Sample of Adult Smokers: Association with Smoking Cessation and Motivation to Quit. Nicotine \& Tobacco research [2014], [ntu200].

doi: $10.1093 / \mathrm{ntr} / \mathrm{ntu} 200$

22. Brown J, Beard E, Kotz D, Michie S, West R: Real-world effectiveness of e-cigarettes when used to aid smoking cessation: a crosssectional population study. Addiction (Abingdon, England) [2014], [109]:[9]:1531-40.

doi: 10.1111/add.12623.
23. Bullen C, Howe C, Laugesen M, McRobbie H, Parag V, Williman J, et al.: Electronic cigarettes for smoking cessation: a randomised controlled trial. Lancet [2013], [382]:[9905]:1629-37.

24. Cahn Z, Siegel M: Electronic cigarettes as a harm reduction strategy for tobacco control: A step forward or a repeat of past mistakes\&quest. Journal of public health policy [2011], [32]:[1]:16-31.

25. Caponnetto P, Campagna D, Cibella F, Morjaria JB, Caruso M, Russo C, et al.: EffiCiency and Safety of an eLectronic cigAreTte (ECLAT) as tobacco cigarettes substitute: a prospective 12-month randomized control design study. PloS one [2013], [8]:[6]:e66317. doi: 10.1371/journal.pone.0066317.

26. Etter JF, Bullen C: A longitudinal study of electronic cigarette users. Addictive behaviors [2013], [39]:[2]:491-4. doi: 10.1016/j.drugalcdep.2016.01.003.

27. Hajek P: Electronic cigarettes have a potential for huge public health benefit. BMC medicine [2014], [12]:[1]:225.

doi: 10.1186/s12916-014-0225-z.

28. Nutt DJ, Phillips LD, Balfour D, Curran HV, Dockrell M, Foulds $\mathrm{J}$, et al.: Estimating the harms of nicotine-containing products using the MCDA approach. European addiction research [2014], [20]:[5]:218-25.

29. Polosa R, Caponnetto P, Morjaria JB, Papale G, Campagna D, Russo C: Effect of an electronic nicotine delivery device (e-Cigarette) on smoking reduction and cessation: a prospective 6-month pilot study. BMC public health [2011], [11]:[786].

doi: 10.1186/1471-2458-11-786.

30. Polosa R, Morjaria JB, Caponnetto P, Campagna D, Russo C, Alamo A, et al.: Effectiveness and tolerability of electronic cigarette in real-life: a 24-month prospective observational study. Internal and emergency medicine [2013], [9]:[5]:537-46. doi: 10.1007/s11739-013-0977-z.

31. Fairchild AL, Bayer R, Colgrove J: The renormalization of smoking? E-cigarettes and the tobacco "endgame". New England Journal of Medicine [2014], [370]:[4]:293-5. doi: 10.1056/NEJMp1313940.

32. Camenga DR, Delmerico J, Kong G, Cavallo D, Hyland A, Cummings $\mathrm{KM}$, et al.: Trends in use of electronic nicotine delivery systems by adolescents. Addictive behaviors [2014], [39]:[1]:338-40. doi: 10.1016/j.addbeh.2013.09.014.

33. Center for Disease Control and Prevention(CDC): Notes from the field: electronic cigarette use among middle and high school students - United States, 2011-2012. MMWR Morbidity and mortality weekly report [2013], [62]:[35]:729-30.

34. Pentz MA, Shin H, Riggs N, Unger JB, Collison KL, Chou CP: Parent, peer, and executive function relationships to early adolescent e-cigarette use: A substance use pathway? Addictive behaviors [2014], [42C]:[73-8]. doi: 10.1016/j.addbeh.2014.10.040.

35. Primack BA, Soneji S, Stoolmiller M, Fine MJ, Sargent JD: Progression to Traditional Cigarette Smoking After Electronic Cigarette Use Among US Adolescents and Young Adults. JAMA pediatrics [2015], [1-7].

doi: 10.1001/jamapediatrics.2015.1742.

36. Dicey J: Shocking new e-cigarette study! The e-cigarette time bomb.... [2014].

37. Berg J: Preferred flavors and reasons for e-cigarette use and discontinued use among never, current, and former smokers. International Journal of Public Health [2016], [61]:[2]:225-236. doi: 10.1007/s00038-015-0764-x. 
38. Kalkhoran S, Grana RA, Neilands TB, Ling PM: Dual use of smokeless tobacco or e-cigarettes with cigarettes and cessation. American Journal of Health Behavior [2015], [39]:[2]:277-284.

39. Chapman SLC, Wu LT: E-cigarette prevalence and correlates of use among adolescents versus adults: A review and comparison. Journal of Psychiatric Research [2014], [54]:43-54. doi: 10.1016/j.jpsychires.2014.03.005.

40. Rüther T, Wissen F, Linhardt A, Aichert DS, Pogarell O, de Vries H: Electronic Cigarettes-Attitudes and Use in Germany. Nicotine \& Tobacco Research [2015], [ntv188].

doi: $10.1093 / \mathrm{ntr} / \mathrm{ntv} 188$.

41. de Vries H, Mesters I, Van de Steeg H, Honing C: The general public's information needs and perceptions regarding hereditary cancer: an application of the Integrated Change Model. Patient education and counseling [2005], [56]:[2]:154-65. doi10.1016/j.pec.2004.01.002.

42. De Vries H, Eggers SM, Bolman C: The role of action planning and plan enactment for smoking cessation. BMC Public Health [2013], [13]:[1]:393. doi: 10.1186/1471-2458-13-393.

43. Eggers SM, Aaro LE, Bos AE, Mathews C, de Vries H: Predicting condom use in South Africa: a test of two integrative models. AIDS and Behavior [2014], [18]:[1]:135-145.

doi: 10.1007/s10461-013-0423-2.

44. Knops-Dullens T, de Vries N, de Vries H: Reasons for nonattendance in cervical cancer screening programmes: an application of the Intergrated Model for Behavioural Change. European Journal of Cancer Prevention [2007], [16]:[5]:436-445. doi: 10.1097/01.cej.0000236250.71113.7c

45. McMillen Trends in Electronic Cigarette Use Among U.S. Adults: Use is Increasing in Both Smokers and Nonsmokers. Nicotine and Tobacco Research [2014].

doi: $10.1093 /$ ntr/ntu213

46. Bunnel RE, Agaku IT, Arrazola RA, Apelberg BJ, Caraballo RS, Corey CG, et al.: Intentions to smoke cigarettes among neversmoking US middle and high school electronic cigarette users: National Youth Tobacco Survey, 2011-2013. Nicotine and Tobacco Research [2015],[17]:228-35. doi: $10.1093 / \mathrm{ntr} / \mathrm{ntu} 166$.

47. Leventhal AM, Strong DR, Kirkpatrick MG, Unger JB, Sussman S, Riggs NR, et al.: Association of electronic cigarette use with initiation of combustile tobacco product smoking in early adolescence. The Journal of American Medical Association [2015],[314]:700-707. doi: 10.1001/jama.2015.8950.

48. Zhu SH, Gamst A, Lee M, Cummins S, Yin L, Zoref L: The Use and Perception of Electronic Cigarettes and Snus among the U.S. Population. PloS one [2013], [8]:[10]:e79332. doi: 10.1371/journal.pone.0079332.

49. Gallus S, Lugo A, Pacifici R, Pichini S, Colombo P, Garattini S, et al.: E-Cigarette Awareness, Use, and Harm Perception in Italy: A National Representative Survey. Nicotine \& Tobacco research [2014], [ntu124]. doi: 10.1093/ntr/ntu124.

50. Kinnunen JM, Ollila H, El-Amin SE, Pere LA, Lindfors PL, Rimpela AH: Awareness and determinants of electronic cigarette use among Finnish adolescents in 2013: a population-based study. Tobacco control [2014], [tobaccocontrol-2013].

doi: 10.1136/tobaccocontrol-2013-051512.
51. McRobbie H, Bullen C, Hajek P: Electronic cigarettes for smoking cessation and reduction. The Cochrane Library [2012], [11].

52. Kalkhoran S, Glantz SA: E-cigarettes and smoking cessation in realworld and clinical settings: a systematic review and meta-analysis. The Lancet Respiratory Medicine [2016], [4]:116-128. doi: 10.1016/S2213-2600(15)00521-4.

53. Pokhrel P, Herzog TA, Muranaka N, Regmi S, Fagan P: Contexts of cigarette and e-cigarette use among dual users: a qualitative study. BMC public health [2015], [15]:[859]. doi: 10.1186/s12889-015-2198-z.
CONFLICT OF INTERESTS The authors have completed and submitted the ICMJE Form for Disclosure of Potential Conflicts of Interest and none were reported.

\section{FUNDING}

This research received no specific grant from any funding agency in the public, commercial, or not-for-profit sectors. This work was financed by CAPHRI, Health Communication, Maastricht University.

\section{PROVENANCE AND PEER} REVIEW

Not commissioned;

Externally peer reviewed 